\title{
Schéma de relaxation pour l'équation de Schrö- dinger non linéaire et les systèmes de Davey et Stewartson
}

\section{Christophe BESSE}

Mathématiques Appliquées de Bordeaux, UPRESA 5466, Université Bordeaux I, 351 Cours de la Libération, 33405 Talence, France (besse@math.u-bordeaux.fr) et Mathématiques pour l'Industrie et la Physique, Université Paul Sabatier, UFR MIG, 118 Route de Narbonne, 31062 Toulouse Cedex 4, France.

Résumé. Dans cette note, nous présentons un nouveau schéma numérique pour l'équation de Schrödinger non linéaire et les systèmes de Davey et Stewartson. C'est un schéma de type relaxation qui supprime la résolution de systèmes non linéaires. Nous donnons des résultats d'existence et de convergence pour une version semi-discrète, localement en temps pour toute donnée initiale et globalement en temps à données petites.

Relaxation scheme for the nonlinear Schrödinger equation and DaveyStewartson systems

\begin{abstract}
In this paper, we introduce a new numerical scheme for the nonlinear Schrödinger equation and the Davey-Stewartson systems. This is a relaxation type scheme that avoids the resolution of nonlinear systems. We give convergence results for the semi-discret version, locally in time for all data and globally in time for small data.
\end{abstract}

Abridged English Version. We introduce a new numerical scheme for the nonlinear Schrödinger equation and the Davey-Stewartson systems which reduces the average time of execution compared with classical numerical schemes for dispersive equations. The aim of this paper is to show the existence of solutions of this scheme and their convergence.

Contrary to the classical Crank-Nicolson scheme [5], we have no nonlinear iteration step. Actually, we suppress the nonlinear part of the scheme by doing a relaxation, which means to separate the treatment of the linear and the nonlinear part to different times. Then, the scheme for the nonlinear Schrödinger equation (1) takes the form (3). In fact, we write the nonlinearity at time $t=n \delta t$ and the linear Schrödinger equation at time $t=\left(n+\frac{1}{2}\right) \delta t$. In a practical point of view, the equation at time $t=n \delta t$ gives the value of $\psi$ at $t=\left(n+\frac{1}{2}\right) \delta t$ that we plug in the second equation, which gives the value of $u$ at 
$t=(n+1) \delta t$.

In a first part, we show the local existence and the uniqueness of a solution for (3) in $H^{s}\left(\mathbb{R}^{d}\right)$, with $s>d / 2+2$. We make use of a fixed point method on a semi linear system (5) equivalent to (3) which takes into account the discrete derivative in time $v^{n+\frac{1}{2}}=\frac{u^{n+1}-u^{n}}{\delta t}$. We are led to define the value of $u^{-1}$ to insure the solution of the third equation of (5) to be bounded in $L^{\infty}\left(0, T ; H^{s}\right)$.

Subsequently, we prove the convergence of the discrete solution to the solution of the continuous (NLS) equation by comparison between the corresponding discrete and continuous Duhamel expressions.

In an other way, we show the global existence of solutions for (3) in $H^{1}\left(\mathbb{R}^{2}\right)$ by making use of a long waves regularization (7) (see [6]). Applying again a fixed point method, we prove local existence of solution. Thanks to conserved quantities, we can globalize and find bounds independent of $\epsilon$ for approximate solution. Then, we can pass to the limit $\epsilon \rightarrow 0$ and conclude to global existence of solution.

In this case, the convergence is made by introduction of auxiliary functions $U_{1}^{\delta t}, U_{2}^{\delta t}$, $U_{3}^{\delta t}, \Psi_{1}^{\delta t}$ and $\Psi_{2}^{\delta t}$ which allow to (3) to be written as a continuous system. Using the bounds found at preceeding step of the proof, we can make use of compactness as in [3].

The same theorems are proved for the Davey-Stewartson version of the numerical scheme (8). All numerical results prove the quality of the scheme. Details of the proofs and several numerical results can be found in $[1,2]$.

1. Introduction Un schéma classique pour l'équation de Schrödinger non linéaire

$$
i u_{t}+L u=\lambda|u|^{2 \sigma} u, x \in \mathbb{R}^{d}, t \geq 0, u(x, 0)=u_{0}(x), x \in \mathbb{R}^{d},
$$

avec $\lambda= \pm 1, \sigma>0$ et $L$ un opérateur du second ordre défini par $L u=\sum_{i, j=1}^{d} a_{i j} \frac{\partial^{2} u}{\partial x_{i} \partial x_{j}}$ où la matrice $\left(a_{i j}\right)$ est symétrique et inversible, est dû à Delfour, Fortin et Payre [5] et prend la forme

$$
i \frac{u^{n+1}-u^{n}}{\delta t}+L\left(\frac{u^{n+1}+u^{n}}{2}\right)=\frac{\lambda}{2 \sigma+2} \frac{\left|u^{n+1}\right|^{2 \sigma+2}+\left|u^{n}\right|^{2 \sigma+2}}{\left|u^{n+1}\right|^{2}-\left|u^{n}\right|^{2}}\left(u^{n+1}+u^{n}\right) .
$$

Ce schéma est très robuste et simple à mettre en œuvre, mais coûte relativement cher en temps de calcul à cause de l'étape non linéaire. Nous proposons un nouveau schéma de relaxation qui supprime le temps d'exécution. Ce schéma s'écrit

$$
\left\{\begin{aligned}
\frac{\psi^{n+\frac{1}{2}}+\psi^{n-\frac{1}{2}}}{2} & =\left|u^{n}\right|^{2 \sigma}, & & x \in \mathbb{R}^{d}, \\
i \frac{u^{n+1}-u^{n}}{\delta t}+L\left(\frac{u^{n+1}+u^{n}}{2}\right) & =\lambda\left(\frac{u^{n+1}+u^{n}}{2}\right) \psi^{n+\frac{1}{2}}, & & x \in \mathbb{R}^{d} .
\end{aligned}\right.
$$

où $u^{0}=u_{0}$ et $\psi^{-\frac{1}{2}}=\psi^{\frac{1}{2}}=\left|u_{0}\right|^{2 \sigma}$. 
2. Existence locale Soit $\left(u^{n}, \psi^{n+\frac{1}{2}}\right)$ vérifiant (3), $u_{\delta t}$ la fonction constante par morceaux qui vaut $u^{n} \operatorname{sur}\left[n \delta t,(n+1) \delta t\left[\right.\right.$, et $\psi_{\delta t}$ qui vaut $\psi^{n+\frac{1}{2}}$ sur l'intervalle $[n \delta t,(n+1) \delta t[$.

Théorème 1 1. Soit $u_{0} \in H^{s}\left(\mathbb{R}^{d}\right), s>d / 2+2$, alors $\exists T_{0}>0$ ne dépendant que de $\left\|u_{0}\right\|_{H^{s}}$ et $\exists T_{\delta t}>T_{0}$ tels que (3) admet une unique solution maximale $\left(u_{\delta t}, \psi_{\delta t}\right)$ dans $L^{\infty}\left(\left[0, T_{\delta t}\right] ;\left(H^{s}\left(\mathbb{R}^{d}\right)\right)^{2}\right)$ avec $T_{\delta t}=N \delta t$.

2. Soit u la solution maximale de (1) définie dans $C^{2}\left(\left[0, T_{-}\left[; H^{s}\right), s>d / 2+4\right.\right.$. Alors, on a $\liminf _{\delta t \rightarrow 0} T_{\delta t} \geq T_{-}$et $\forall T<T_{-}$, si $\delta t$ est assez petit, $\left(u_{\delta t}, \psi_{\delta t}\right) \in\left(L^{\infty}\left([0, T] ; H^{s}\right)\right)^{2}$ et $\left(u_{\delta t}, \psi_{\delta t}\right) \longrightarrow\left(u,|u|^{2}\right)$ dans $\left(L^{\infty}\left([0, T] ; H^{s}\right)\right)^{2}$ quand $\delta t \rightarrow 0$.

\section{Schéma de la preuve du théorème (1)}

Afin de simplifier la démonstration, nous supposons $\lambda=1$ et $\sigma=1$. La première équation de (3) s'écrit

$$
\frac{\psi^{n+\frac{1}{2}}+\psi^{n-\frac{1}{2}}}{2}=\left|u^{n}\right|^{2} .
$$

En réécrivant (4) au temps précédent $(n-1)$, en la soustrayant de (4) et en la divisant par $\delta t$, nous avons $\frac{\psi^{n+\frac{1}{2}}-\psi^{n-\frac{3}{2}}}{2 \delta t}=\frac{\left|u^{n}\right|^{2}-\left|u^{n-1}\right|^{2}}{\delta t}$. Nous obtenons donc une équation d'évolution $\operatorname{sur} \psi^{n+\frac{1}{2}}$. Cependant, le membre de droite de cette équation est $\frac{\left|u^{n}\right|^{2}-\left|u^{n-1}\right|^{2}}{\delta t}$. Il n'est donc pas possible d'appliquer directement une procédure de point fixe à ce système à cause soit de la perte d'uniformité en $\delta t$, soit de la perte de régularité si nous remplaçons $\frac{\left|u^{n}\right|^{2}-\left|u^{n-1}\right|^{2}}{\delta t}$ par $\operatorname{Im}\left(L\left(\frac{u^{n+1}+u^{n}}{2}\right)\left(\overline{u^{n+1}+u^{n}}\right)\right)$. L'idée est de prendre $v^{n+\frac{1}{2}}=\frac{u^{n+1}-u^{n}}{\delta t}$ comme nouvelle variable indépendante. Nous obtenons alors un nouveau système équivalent à (3) et qui s'écrit

$$
\left\{\begin{array}{l}
\frac{u^{n+1}-u^{n}}{\delta t}-i L\left(\frac{u^{n+1}+u^{n}}{2}\right)=-i g^{n+\frac{1}{2}} \\
\frac{\psi^{n+\frac{3}{2}}-\psi^{n-\frac{1}{2}}}{2 \delta t}=\Lambda^{n+\frac{1}{2}} \\
\frac{v^{n+\frac{3}{2}}-v^{n-\frac{1}{2}}}{2 \delta t}-i L\left(\frac{v^{n+\frac{3}{2}}+2 v^{n+\frac{1}{2}}+v^{n-\frac{1}{2}}}{4}\right)=-i \Upsilon^{n+\frac{1}{2}}
\end{array}\right.
$$

avec les données initiales $u^{0}, \psi^{\frac{1}{2}}=\psi^{-\frac{1}{2}}=\left|u^{0}\right|^{2 \sigma}, v^{\frac{1}{2}}=\frac{u^{1}-u^{0}}{\delta t}$ et $v^{-\frac{1}{2}}=\frac{u^{0}-u^{-1}}{\delta t}$, où $g^{n+\frac{1}{2}}=$ $\psi^{n+\frac{1}{2}}\left(\frac{u^{n+1}+u^{n}}{2}\right), \Lambda^{n+\frac{1}{2}}=2 \operatorname{Re}\left[v^{n+\frac{1}{2}}\left(\frac{\overline{u^{n+1}+u^{n}}}{2}\right)\right]$ et $\Upsilon^{n+\frac{1}{2}}=\left(\frac{\psi^{n+\frac{3}{2}}+\psi^{n-\frac{1}{2}}}{2}\right)\left(\frac{v^{n+\frac{3}{2}}+2 v^{n+\frac{1}{2}}+v^{n-\frac{1}{2}}}{4}\right)+$ $2 \operatorname{Re}\left[v^{n+\frac{1}{2}}\left(\overline{\frac{u^{n+1}+u^{n}}{2}}\right)\right]\left(\frac{u^{n+2}+u^{n+1}+u^{n}+u^{n-1}}{4}\right)$. On pose $A=\left(I-i \frac{\delta t L}{2}\right)^{-1}\left(I+i \frac{\delta t}{2}\right)$, c'est le générateur associé à la partie linéaire de $(3)$, i.e.

$$
i \frac{u^{n+1}-u^{n}}{\delta t}+L\left(\frac{u^{n+1}+u^{n}}{2}\right)=0 \Leftrightarrow u^{n+1}=A u^{n} .
$$

Nous définissons alors $u^{-1}$ par $A u^{-1}=(A+1) u^{0}-u^{1}$. Le choix de $u^{-1}$ n'est pas arbitraire. C'est en effet le seul choix qui nous assure que pour $v^{\frac{1}{2}}$ et $v^{-\frac{1}{2}}$ définis ci-dessus, la solution de $\frac{v^{n+\frac{3}{2}}-v^{n-\frac{1}{2}}}{2 \delta t}-i L \frac{v^{n+\frac{3}{2}}+2 v^{n+\frac{1}{2}}+v^{n-\frac{1}{2}}}{4}=f^{n+\frac{1}{2}}$ est bornée dans $L^{\infty}\left(0, T ; H^{s}\right)$ dès que $\left(f^{n+\frac{1}{2}}\right)_{n}$ est bornée dans $L^{1}\left(0, T ; H^{s}\right)$. Le système $(5)$ est semi-linéaire et nous appliquons classiquement une méthode de point fixe. 
Pour montrer la convergence du schéma, on procède comme dans [4] et on écrit les formules de Duhamel discrètes issues de (5) et les formules continues issues du système continu correspondant

$$
\begin{aligned}
& u(x, t)=S(t) u(x, t=0)-i \int_{0}^{t} S(t-s)|u|^{2} u(x, s) d s, x \in \mathbb{R}^{d}, t>0, \\
& \psi(x, t)=\psi(x, t=0)+\int_{0}^{t} 2 \operatorname{Re}(\bar{u} v)(x, s) d s, x \in \mathbb{R}^{d}, t>0 \\
& v(x, t)=S(t) v(x, t=0)-i \int_{0}^{t} S(t-s) \Upsilon(x, s) d s, x \in \mathbb{R}^{d}, t>0
\end{aligned}
$$

où $S(t)$ désigne le groupe de Schrödinger $S(t)=\exp (i \Delta t), v=u_{t}$ et $\Upsilon(x, \tau)=(v \psi+$ $2 \operatorname{Re}(v \bar{u}) u)(x, \tau)$. On construit alors à partir de $u^{n}, \psi^{n+\frac{1}{2}}, v^{n+\frac{1}{2}}$ des fonctions $C^{0}$ par morceaux $u_{\delta t}, \psi_{\delta t}, v_{\delta t}$ comme pour l'existence. Comme le problème de Cauchy pour (5) et (6) est résolu par une méthode de contraction, on obtient une estimation du type $\left\|u_{\delta t}-u\right\|_{L^{\infty}\left(0, T ; H^{s}\right)} \stackrel{\delta t \rightarrow 0}{\longrightarrow} 0$ (voir $[1,2]$ pour les détails).

3. Existence globale Dans le cas où $L=\Delta, \sigma=1$ et $\lambda=-1$, nous avons un résultat global.

Théorème 2 1. On suppose $L=\Delta, \sigma=1, \lambda=-1$. Pour $u^{0} \in H^{1}\left(\mathbb{R}^{2}\right)$ telle que $\left\|u^{0}\right\|_{H^{1}\left(\mathbb{R}^{2}\right)}<1$, le système $(3)$ admet une solution $\left(U^{n}, \Psi^{n-\frac{1}{2}}\right) \in l^{\infty}\left(\mathbb{N} ; H^{1}\left(\mathbb{R}^{2}\right) \times\right.$ $\left.L^{2}\left(\mathbb{R}^{2}\right)\right)$.

2. Si $\delta t$ est suffisament petit, on a $u_{\delta t} \stackrel{\delta t \rightarrow 0}{\longrightarrow} u$ dans $L_{l o c}^{2}\left(\mathbb{R}^{+} ; L_{l o c}^{2}\left(\mathbb{R}^{2}\right)\right)$ fortement et $\psi_{\delta t} \stackrel{\delta t \rightarrow 0}{\rightarrow} \psi$ dans $L_{\text {loc }}^{2}\left(\mathbb{R}^{+} ; L_{\text {loc }}^{2}\left(\mathbb{R}^{2}\right)\right)$ faiblement. De plus, $(u, \psi)$ satisfont

$$
\begin{cases}i u_{t}+\Delta u=-u \psi, & x \in \mathbb{R}^{2}, \\ \psi=|u|^{2}, & x \in \mathbb{R}^{2}\end{cases}
$$

\section{Schéma de la preuve du théorème 2}

La preuve repose sur le fait que (3) possède des invariants qui s'écrivent dans ce cas :

Lemme 1 Supposons que $\left(u^{n}\right)_{n \in[0, N]} \in\left(H^{1}\left(\mathbb{R}^{d}\right)\right)^{N+1}$ et $\left(\psi^{n+\frac{1}{2}}\right)_{n=[-1, N]} \in\left(L^{2}\left(\mathbb{R}^{d}\right)\right)^{N+2}$ sont solutions de (3), alors, $\forall n \leq N \int_{\mathbb{R}^{d}}\left|u^{n}\right|^{2} d x=\int_{\mathbb{R}^{d}}\left|u^{0}\right|^{2} d x$, et

$$
\int_{\mathbb{R}^{d}}\left|\nabla u^{n}\right|^{2}+\lambda\left|u^{n}\right|^{2} \psi^{n-\frac{1}{2}}-\lambda \frac{\left(\psi^{n-\frac{1}{2}}\right)^{2}}{2} d x=\int_{\mathbb{R}^{d}}\left|\nabla u^{0}\right|^{2}+\lambda\left|u^{0}\right|^{2} \psi^{-\frac{1}{2}}-\lambda \frac{\left(\psi^{-\frac{1}{2}}\right)^{2}}{2} d x .
$$

Nous effectuons alors une régularisation ondes longues inspirée par [6]. Le système perturbé s'écrit

$$
\left\{\begin{aligned}
i \frac{u_{\epsilon}^{n+1}-u_{\epsilon}^{n}}{\delta t}+\Delta\left(\frac{u_{\epsilon}^{n+1}+u_{\epsilon}^{n}}{2}\right)-i \epsilon \Delta\left(\frac{u_{\epsilon}^{n+1}-u_{\epsilon}^{n}}{\delta t}\right) & =-\left(\frac{u_{\epsilon}^{n+1}+u_{\epsilon}^{n}}{2}\right) \psi_{\epsilon}^{n+\frac{1}{2}} \\
\frac{\psi_{\epsilon}^{n+\frac{1}{2}}+\psi_{\epsilon}^{n-\frac{1}{2}}}{2} & =\left|u_{\epsilon}^{n}\right|^{2}
\end{aligned}\right.
$$

Il est facile de montrer à l'aide d'une méthode de point fixe l'existence locale de solutions de (7). En utilisant l'équivalent du lemme 1 pour (7), nous pouvons globaliser et trouver 
des bornes indépendantes de $\epsilon$ en supposant la norme $H^{1}$ de la donnée initiale assez petite. En extrayant des sous-suites, et en utilisant de la compacité, nous pouvons passer à la limite en $\epsilon$. Pour la convergence du schéma, on introduit des fonctions auxiliaires. Soit $U_{1}^{\delta t}$ la fonction affine par morceaux qui vaut $u^{l}$ à $t=l \delta t$. De même, définissons $U_{2}^{\delta t}$, $U_{3}^{\delta t}, \Psi_{1}^{\delta t}$ et $\Psi_{2}^{\delta t}$ qui valent respectivement $\frac{u^{l}+u^{l-1}}{2}, u^{l-1}, \psi^{l-\frac{1}{2}}$ et $\frac{\psi^{l-\frac{1}{2}}+\psi^{l-\frac{3}{2}}}{2}$ sur l'intervalle $[(l-1) \delta t, l \delta t[$. Avec ces notations, en posant $\delta t=T /(n-1),(3)$ s'écrit exactement

$$
i \partial_{t} U_{1}^{\delta t}+\Delta U_{2}^{\delta t}=-U_{2}^{\delta t} \Psi_{1}^{\delta t}, \Psi_{2}^{\delta t}=\left|U_{3}^{\delta t}\right|^{2} .
$$

Comme dans [6], on montre en utilisant le lemme que si $\left\|u_{0}\right\|_{H^{1}}<1$, la suite $\left(u^{k}\right)_{k \in[0, n-1]}$ est bornée dans $l^{\infty}\left(0, n-1 ; H^{1}\left(\mathbb{R}^{2}\right)\right)$ et donc les termes $U_{1}^{\delta t}, U_{2}^{\delta t}$ et $U_{3}^{\delta t}$ sont bornées dans $L^{\infty}\left(0, T ; H^{1}\left(\mathbb{R}^{2}\right)\right)$. De la même manière, les suites $\Psi_{1}^{\delta t}$ and $\Psi_{2}^{\delta t}$ sont bornées dans $L^{\infty}\left(0, T ; L^{2}\left(\mathbb{R}^{2}\right)\right)$. De plus, on a $\partial_{t} U_{1}^{\delta t}=i \Delta U_{2}^{\delta t}+i U_{2}^{\delta t} \Psi_{1}^{\delta t}$. Ainsi, $\partial_{t} U_{1}^{\delta t}(t)$ est borné dans $L^{\infty}\left(0, T ; H^{-1}\left(\mathbb{R}^{2}\right)\right)$ et donc $U_{1}^{\delta t}$ converge dans $L_{\text {loc }}^{\infty}\left(\mathbb{R} ; L_{\text {loc }}^{2}\left(\mathbb{R}^{2}\right)\right)$ fort et dans $L^{\infty}\left(\mathbb{R} ; H^{1}\left(\mathbb{R}^{2}\right)\right)$ faible vers $U(t)$. En outre, $\Psi_{1}^{\delta t}$ (resp. $\left.\Psi_{2}^{\delta t}\right)$ converge dans $L^{\infty}\left(0, T ; L^{2}\left(\mathbb{R}^{2}\right)\right)$ faible vers $\Psi_{1}(t)$ (resp. $\Psi_{2}(t)$ ). En utilisant les mêmes techniques que dans [3], nous montrons

Lemme 2 Les suites $U_{2}^{\delta t}(t)$ et $U_{3}^{\delta t}(t)$ convergent fort vers $U(t)$ dans $L^{2}\left(0, T ; L^{2}\left(\mathbb{R}^{2}\right)\right)$. De plus, $\lim _{\delta t \rightarrow 0}\left\|\Psi_{2}^{\delta t}-\Psi_{1}^{\delta t}\right\|_{L^{2}\left(0, T ; H^{-3}\right)}=0$.

Grâce à toutes ces estimations, nous pouvons passer à la limite dans (3).

4. Cas de DS Les systèmes de Davey-Stewartson s'écrivent sous la forme (voir [6])

$$
i u_{t}+u_{x x}+\epsilon u_{y y}=\chi|u|^{2} u+u \varphi_{x}, \varphi_{x x}+m \varphi_{y y}=|u|_{x}^{2}, \epsilon= \pm 1, m= \pm 1, \chi \in \mathbb{R} .
$$

Notre schéma de relaxation s'écrit :

$$
\begin{cases}i \frac{u^{n+1}-u^{n}}{\delta t}+A\left(\frac{u^{n+1}+u^{n}}{2}\right)=\left[\chi \psi^{n+\frac{1}{2}}+\lambda \varphi_{x}^{n+\frac{1}{2}}\right]\left(\frac{u^{n+1}+u^{n}}{2}\right), & (x, y) \in \mathbb{R}^{2}, \\ B\left(\frac{\varphi^{n+\frac{1}{2}}+\varphi^{n-\frac{1}{2}}}{2}\right)=\left(\left|u^{n}\right|^{2}\right)_{x}, \quad \frac{\psi^{n+\frac{1}{2}}+\psi^{n-\frac{1}{2}}}{2}=\left|u^{n}\right|^{2}, \quad(x, y) \in \mathbb{R}^{2},\end{cases}
$$

avec $A=\partial_{x x}+\epsilon \partial_{y y}$ et $B=\partial_{x x}+m \partial_{y y}$. Dans le cas où $m=+1$, le théorème 1 s'adapte

Théorème 3 1. Soit $u_{0} \in H^{s}\left(\mathbb{R}^{2}\right), s>3$, alors $\exists T_{0}>0$ ne dépendant que de $\left\|u_{0}\right\|_{H^{s}}$ et $\exists T_{\delta t}>T_{0}$ tels que (8) admet une unique solution maximale $\left(u_{\delta t}, \varphi_{\delta t}, \psi_{\delta t}\right)$ dans $L^{\infty}\left(\left[0, T_{\delta t}\right] ;\left(H^{s}\left(\mathbb{R}^{2}\right)\right)^{3}\right)$ avec $T_{\delta t}=N \delta t$.

2. Soit u la solution maximale de $(D S)$ définie dans $C^{2}\left(\left[0, T_{-}\left[; H^{s}\right), s>5\right.\right.$. Alors, on a $\liminf _{\delta t \rightarrow 0} T_{\delta t} \geq T_{-}$et $\forall T<T_{-}$, si $\delta t$ est assez petit, $\left(u_{\delta t}, \varphi_{\delta t}, \psi_{\delta t}\right) \in\left(L^{\infty}\left([0, T] ; H^{s}\right)\right)^{3}$ et $\left(u_{\delta t}, \varphi_{\delta t}, \psi_{\delta t}\right) \longrightarrow\left(u, \varphi,|u|^{2}\right)$ dans $\left(L^{\infty}\left([0, T] ; H^{s}\right)\right)^{3}$ quand $\delta t \rightarrow 0$.

Dans le cas où $m=+1$ et $\epsilon=+1$, le théorème 2 s'adapte :

Théorème 4 1. On suppose $\chi=-1$. Pour $u^{0} \in H^{1}\left(\mathbb{R}^{2}\right)$ telle que $\left\|u^{0}\right\|_{H^{1}\left(\mathbb{R}^{2}\right)}<1$, le système (8) admet une solution $\left(U^{n}, \varphi^{n-\frac{1}{2}}, \Psi^{n-\frac{1}{2}}\right) \in l^{\infty}\left(\mathbb{N} ; H^{1}\left(\mathbb{R}^{2}\right) \times H^{1}\left(\mathbb{R}^{2}\right) \times\right.$ $\left.L^{2}\left(\mathbb{R}^{2}\right)\right)$. 
2. Si $\delta t$ est suffisament petit, on a $u_{\delta t} \stackrel{\delta t \rightarrow 0}{\longrightarrow} u$ dans $L_{\text {loc }}^{2}\left(\mathbb{R}^{+} ; L_{\text {loc }}^{2}\left(\mathbb{R}^{2}\right)\right)$ fortement,

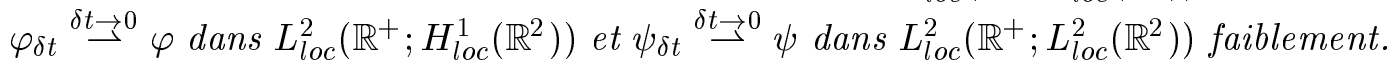
De plus, $(u, \varphi, \psi)$ satisfont

$$
\begin{cases}i u_{t}+\Delta u=-u \psi+u \varphi_{x}, & x \in \mathbb{R}^{2}, \\ \Delta \varphi=|u|_{x}^{2}, & x \in \mathbb{R}^{2}, \\ \psi=|u|^{2}, & x \in \mathbb{R}^{2} .\end{cases}
$$

Les preuves sont sensiblement les mêmes (voir $[1,2]$ ).

5. Tests numériques Les résultats numériques sont tous bons (voir [1, 2]) et les gains en temps de calcul significatifs comme on peut le constater sur la table ci-dessous qui compare les temps de calculs en secondes entre le schéma de Crank-Nicolson (2) et le schéma (3) en fonction du nombre de mailles $N g$, appliqués à l'équation de Schrödinger focalisante $1 \mathrm{D}$ avec $\delta t=10^{-3}$ et 2000 itérations en temps.

\begin{tabular}{|c||c||c|}
\hline $\mathrm{Ng}$ & $(2)$ & $(3)$ \\
\hline 128 & 6.95 & 1.1 \\
256 & 15.15 & 2.64 \\
512 & 31.45 & 6.14 \\
1024 & 64.56 & 12.65 \\
2048 & 130.07 & 25.92 \\
\hline
\end{tabular}

Table 1: Comparaison des deux schémas

Les démonstrations complètes et de nombreux tests numériques se trouvent dans $[1,2]$.

\section{Références bibliographiques}

[1] Besse C. Analyse numérique des systèmes de Davey-Stewartson, Thèse Université Bordeaux I, 1998.

[2] Besse C. Relaxation schemes for nonlinear Schrödinger equations and Davey-Stewartson systems, Rapport Interne 97031, Mathématiques Appliquées de Bordeaux, 1997.

[3] Colin T. The cauchy problem and the continuous limit for the multilayer model in geophysical fluid dynamics. SIAM J. Math. Anal, 28(3):516-529, 1997.

[4] Colin T. and Fabrie P. Semidiscretization in time for nonlinear Schrödinger-waves equations, A paraître dans Discrete and continuous dynamical systems, 1998.

[5] Delfour M., Fortin M. and Payre G. Finite-difference solutions of a non-linear Schrödinger equation, Journal of computational physics, vol 44, pages 277-288, 1981.

[6] Ghidaglia J-M. and Saut J-C. On the initial value problem for the Davey-Stewartson systems, Nonlinearity 3, pages 475-506, 1990. 